\title{
The Method of Linear Equations for Nonzero Temperatures in One-Dimensional Ising Models
}

\author{
MARIAN BŁASZYK \\ Institute of Physics, A. Mickiewicz University \\ Umultowska 85, 61-614 Poznań, Poland
}

(Received December 2, 2004)

\begin{abstract}
The method of linear equations was applied to nonzero temperatures. Two models were considered. The first is the disordered model of random ferromagnetic and antiferromagnetic integrals whose transition matrix meets the condition of invariance of the sum of terms in each line. Although the other model (one-dimensional Ising model in an external field) is devoid of disorder it does not require any assumptions on the form of the transition matrix.
\end{abstract}

PACS numbers: 05.50.+q, 75.10.Nr, 75.50.Lk

\section{Introduction}

The paper is a continuation of the earlier study [1] presenting the method of linear equations permitting a reduction of the physical problem of thermodynamical quantities determination for the systems with frozen disorder to the mathematical problem of solving a set of linear equations. A natural consequence was the question if it is possible to apply this method to the systems at nonzero temperatures. This is not a problem of a simple generalisation but its solution would respond to a great demand as, although the disordered systems have been studied for a few decades no exact results are available even for the simplest systems, besides the ground state. A possible reason is that the calculations are performed on the partition function which means that all details of the exchange integrals are important. In the linear equation method proposed the calculation is performed on the probabilities, which simplifies the description of the problem 
and the calculation procedure. Moreover, the method of linear equations requires consideration of a small fragment of the infinite system whose thermodynamical quantities are averaged.

This paper is composed of two parts dealing with the application of the linear equation method to two models at nonzero temperature. Section 2 presents the application of the method of linear equations to a one-dimensional Ising model with random ferro- and antiferromagnetic integrals on the square lattice rolled on a tube. For this system the temperature dependent internal energy and entropy were found. In Sec. 3 the method of linear equations is applied to one-dimensional Ising ferromagnet in an external magnetic field. The study is summed up in Sec. 4.

\section{The Ising model $\pm J$}

The method of linear equations has been proposed in order to determine the ground state properties of systems with randomly distributed integrals $\pm J$ [1]. In this method the steady state value of the vector of state $\boldsymbol{\pi}$ described by the equation

$$
Q \cdot \pi=\pi
$$

is determined. The vector $\boldsymbol{\pi}$ is made of the vectors $\boldsymbol{P}_{i}$ corresponding to different possible excitations in a base of ferro- and antiferromagnetic integrals. Since the coordinates of $\boldsymbol{P}_{i}$ correspond to the probabilities of the mutually exclusive events the sum $\sum_{i} \boldsymbol{P}_{i}=\boldsymbol{J} \boldsymbol{e}$, where $\boldsymbol{J} \boldsymbol{e}$ is the vector whose coordinates are all equal to 1 . The matrix $\boldsymbol{Q}$ is made of the matrices $\boldsymbol{M}_{i}$ describing the probabilities of all possible sequences of frustrated and unfrustrated squares and meeting the condition $\boldsymbol{M} \cdot \boldsymbol{J} \boldsymbol{e}=\boldsymbol{J} \boldsymbol{e}$, where $\boldsymbol{M}=\sum_{i} \boldsymbol{M}_{i}$. The condition defines the Markov process and will be referred to as the Markov condition or matrix, and it is usually written in the form: $\sum_{l} \boldsymbol{M}(k, l)=1$. This condition implies that the probability of finding the ferromagnetic bond is $1-p$, whereas that of finding the antiferromagnetic bond is $p$ and the probability of finding either one or the other is a certain event of the probability 1. Let us consider now if it is possible and if yes - in which way to apply the method to a system with averaging over the canonical ensemble, when the probability of finding a certain spin state is directly proportional to the Boltzmann factor. It could be supposed that by replacing the factor $\mathrm{e}^{\beta}$ by $\mathrm{e}^{\beta} /\left(\mathrm{e}^{\beta}+\mathrm{e}^{-\beta}\right)$ for the satisfied bond (of lower energy) and the factor $\mathrm{e}^{-\beta}$ by $\mathrm{e}^{-\beta} /\left(\mathrm{e}^{\beta}+\mathrm{e}^{-\beta}\right)$ for the unsatisfied bond it would be possible to meet the Markov condition so to a possibility of applying the method of linear equations. However, it is not so as the replacement of the probabilistic weights $(p, 1-p)$ by $\left(\mathrm{e}^{\beta}, \mathrm{e}^{-\beta}\right)$ is not the only difference between the averaging over disorder and averaging over the canonical ensemble. If we average over disorder, the exchange integrals assume the values of $+J$ and $-J$ independently of each other, but the averaging over the canonical ensemble is performed for a certain distribution of the exchange integrals and a 
different condition is imposed on the satisfied and unsatisfied bonds when the elementary polygon is frustrated and unfrustrated. The square lattice considered can only have unfrustrated squares without, with two or with four unsatisfied bonds and frustrated squares with one or three unsatisfied bonds. These restrictions violate the Markov condition and change the condition for the stationary probability distribution from Eq. (1) into

$$
\boldsymbol{Q} \cdot \boldsymbol{\pi}=\lambda \boldsymbol{\pi},
$$

where $\lambda$ is the factor reducing the matrix $\boldsymbol{Q}$ to the Markov form defined by the relation $\sum_{j} \boldsymbol{M}(i, j)=\lambda$ and as follows from (2) it is an eigenvalue of the matrix $\boldsymbol{Q}$.

In order to illustrate the method let us consider an infinite tube made of squares of one bond in width. It is a one-dimensional model with additional bonds perpendicular to the tube. When these bonds are ferromagnetic they are satisfied and when they are antiferromagnetic they are unsatisfied. The system is described by the Hamiltonian

$$
\mathcal{H}=-\sum_{i, j} J_{i j} S_{i} S_{j},
$$

where $(i j)$ refer to the pairs of neighbouring spins, $J_{i j}=J$ with the probability $1-p$ and $J_{i j}=-J$ with the probability $p$. The vector of state is selected in a natural way: its first coordinate is the probability that the spin is directed upwards $P(\uparrow)$ while the second coordinate is the probability that it is directed downwards $P(\downarrow)=1-P(\uparrow)$. It has been shown that the eigenvector for each choice of the exchange integrals is the same and the matrices of transition commute. It is a consequence of the fact that the thermodynamical quantities depend only on the concentration of the antiferromagnetic integrals $p$ and not on their distribution between the bonds perpendicular or parallel to the tube. As follows from the above, each positioning of the exchange integrals can be considered independently.

The eigenequation for the case when both bonds are ferromagnetic can be written as

$$
\left[\begin{array}{cc}
\mathrm{e}^{2 K} & 1 \\
1 & \mathrm{e}^{2 K}
\end{array}\right]\left[\begin{array}{c}
P(\uparrow) \\
P(\downarrow)
\end{array}\right]=\lambda_{\mathrm{ff}}\left[\begin{array}{c}
P(\uparrow) \\
P(\downarrow)
\end{array}\right]
$$

where $\lambda_{\mathrm{ff}}=\mathrm{e}^{2 K} \pm 1$ and $K=\beta J$. However, for $\lambda_{\mathrm{ff}}=\mathrm{e}^{2 K}-1, P(\uparrow) \notin\langle 0,1\rangle$ so the lower value should be rejected. If $\lambda_{\mathrm{ff}}=\mathrm{e}^{2 K}+1$ then $P(\uparrow)=P(\downarrow)=1 / 2$. The choice of a higher value is also imposed by the Markov condition. This condition is satisfied by the matrix divided by the higher eigenvalue as the sum of terms in both lines is the same and equals $\mathrm{e}^{2 K}+1$. When the ferromagnetic bond is perpendicular to the tube and the antiferromagnetic one is along the tube, then 


$$
\left[\begin{array}{cc}
1 & \mathrm{e}^{2 K} \\
\mathrm{e}^{2 K} & 1
\end{array}\right]\left[\begin{array}{c}
P(\uparrow) \\
P(\downarrow)
\end{array}\right]=\lambda_{\mathrm{fa}}\left[\begin{array}{c}
P(\uparrow) \\
P(\downarrow)
\end{array}\right]
$$

Again the probability of the physical meaning $P(\uparrow)=P(\downarrow)=1 / 2$ corresponds to the higher eigenvalue of $\lambda_{\mathrm{fa}}=\mathrm{e}^{2 K}+1$. The exchange of the ferromagnetic and antiferromagnetic bonds leads to the following equation:

$$
\left[\begin{array}{cc}
1 & \mathrm{e}^{-2 K} \\
\mathrm{e}^{-2 K} & 1
\end{array}\right]\left[\begin{array}{c}
P(\uparrow) \\
P(\downarrow)
\end{array}\right]=\lambda_{\mathrm{af}}\left[\begin{array}{c}
P(\uparrow) \\
P(\downarrow)
\end{array}\right]
$$

and only the higher eigenvalue $\lambda_{\mathrm{af}}=1+\mathrm{e}^{-2 K}$ gives a solution of physical sense $P(\uparrow)=P(\downarrow)=1 / 2$. We get the same solution when both bonds are antiferromagnetic so for $\lambda_{\text {aa }}=1+\mathrm{e}^{-2 K}$ and $P(\uparrow)=P(\downarrow)=1 / 2$. As for each of the cases we have the same vector of state, the value $-\beta F$ averaged over the distribution of the exchange integrals can be written as

$$
\begin{aligned}
& \langle-\beta F\rangle_{J}=(1-p)^{2} \ln \lambda_{\mathrm{ff}}+(1-p) p \ln \lambda_{\mathrm{fa}}+p(1-p) \ln \lambda_{\mathrm{af}}+p^{2} \ln \lambda_{\mathrm{aa}} \\
& =(1-p) \ln \left(\mathrm{e}^{2 K}+1\right)+p \ln \left(\mathrm{e}^{-2 K}+1\right) .
\end{aligned}
$$

Having determined the above value we are able to find the internal energy

$$
\langle E\rangle_{J}=\frac{\partial}{\partial \beta}\langle\beta F\rangle_{J}=2 J\left(p-\frac{1}{1+\mathrm{e}^{-2 K}}\right)
$$

and the value of entropy is already independent of the concentration of the exchange integrals.

$$
\langle S / k\rangle_{J}=\ln 2+\ln \cosh K-K \tanh K .
$$

The model considered above is so simplified that it has lost some essential features of an disordered system. In less simplified cases we have a lot of vectors of state and the relationships between them imply the necessity of considering an infinite number of the vectors with the arbitrary probability $P(\uparrow)$. However, this is not the main obstacle in application of this method. The above described method is limited only to the systems whose transition matrix can be reduced to the Markov matrix and for which $\frac{1}{\lambda} \sum_{j} \boldsymbol{Q}(i, j)=1$, where $\lambda$ is the highest eigenvalue of the matrix $\boldsymbol{Q}$. In order to generalise this method over other matrices we shall consider an ordered one-dimensional Ising model.

\section{The one-dimensional ferromagnetic Ising model in uniform magnetic field}

Let us consider the Ising model described with the Hamiltonian

$$
\mathcal{H}=-J \sum_{i} S_{i} S_{i+1}-H \sum_{i} S_{i}
$$

with $S_{i}= \pm 1$. Denoting $K=\beta J, B=\beta H$, similarly as above we can write the 
eigenequation for the transition matrix in the form

$$
\left[\begin{array}{cc}
\mathrm{e}^{K+B} & \mathrm{e}^{-K} \\
\mathrm{e}^{-K} & \mathrm{e}^{K-B}
\end{array}\right]\left[\begin{array}{l}
\psi(\uparrow) \\
\psi(\downarrow)
\end{array}\right]=\lambda\left[\begin{array}{c}
\psi(\uparrow) \\
\psi(\downarrow)
\end{array}\right] .
$$

Although this equation can be interpreted as the condition of the vector of state preservation, we cannot treat $\psi(\uparrow)$ as the probability of the spin being directed upwards and $\psi(\downarrow)$ as the probability of the spin being directed downwards because the matrix does not satisfy the Markov condition. The relation with probability is obtained by imposing the normalisation condition of the form

$$
\left[\begin{array}{ll}
\psi(\uparrow) & \psi(\downarrow)
\end{array}\right]\left[\begin{array}{l}
\psi(\uparrow) \\
\psi(\downarrow)
\end{array}\right]=1
$$

onto the vector state. Equation (11) can be rewritten as

$$
\left[\begin{array}{ll}
\psi(\uparrow) & \psi(\downarrow)
\end{array}\right]\left[\begin{array}{cc}
\mathrm{e}^{K+B} / \lambda & \mathrm{e}^{-K} / \lambda \\
\mathrm{e}^{-K} / \lambda & \mathrm{e}^{K-B} / \lambda
\end{array}\right]\left[\begin{array}{l}
\psi(\uparrow) \\
\psi(\downarrow)
\end{array}\right]=1 .
$$

As the vector of state is defined to the accuracy of a phase factor, similarly as in the quantum mechanics, Eq. (13) can be rewritten as

$$
\left[|\psi(\uparrow)| \mathrm{e}^{-\mathrm{i} \alpha} \quad|\psi(\downarrow)| \mathrm{e}^{-\mathrm{i} \alpha}\right]\left[\begin{array}{cc}
\mathrm{e}^{K+B} / \lambda & \mathrm{e}^{-K} / \lambda \\
\mathrm{e}^{-K} / \lambda & \mathrm{e}^{K-B} / \lambda
\end{array}\right]\left[\begin{array}{c}
|\psi(\uparrow)| \mathrm{e}^{\mathrm{i} \alpha} \\
|\psi(\downarrow)| \mathrm{e}^{\mathrm{i} \alpha}
\end{array}\right]=1
$$

or in a shortened form

$$
\langle\boldsymbol{\psi}|\boldsymbol{F} / \lambda| \boldsymbol{\psi}\rangle=1,
$$

where $\boldsymbol{F}$ is the transition matrix for the ferromagnetic bond. As all the terms of the matrix are real, the phase factor can be omitted in the following if we consider one of the states. However, when considering many states its presence must be taken into regard in order to ensure orthonormalisation of the state vectors corresponding to different eigenvalues. In the case considered we have two eigenvalues: $\lambda_{ \pm}=$ $\mathrm{e}^{K}\left(\cosh B \pm \sqrt{\sinh ^{2} B+\mathrm{e}^{-4 K}}\right)$, corresponding to two eigenvectors $\left\langle\boldsymbol{\psi}_{+}\right|$and $\left\langle\boldsymbol{\psi}_{-}\right|$ of the terms $\psi_{+}(\uparrow)=\sqrt{\frac{1}{2}+\frac{1}{2}\left\langle s_{i}\right\rangle}, \psi_{+}(\downarrow)=\sqrt{\frac{1}{2}-\frac{1}{2}\left\langle s_{i}\right\rangle}, \psi_{-}(\uparrow)=\sqrt{\frac{1}{2}-\frac{1}{2}\left\langle s_{i}\right\rangle}$, $\psi_{-}(\downarrow)=-\sqrt{\frac{1}{2}+\frac{1}{2}\left\langle s_{i}\right\rangle}$, where $\left\langle s_{i}\right\rangle=\frac{\sinh B}{\sqrt{\sinh ^{2} B+\mathrm{e}^{-4 K}}}$ is the mean value of the spin of the infinite system. This expression has been imposed by the normalisation condition as $\left|\psi_{+}(\uparrow)\right|^{2}=\frac{1}{2}\left(1+\left\langle s_{i}\right\rangle\right)$ is the probability that the spin is directed upwards and $\left|\psi_{+}(\downarrow)\right|^{2}=\frac{1}{2}\left(1-\left\langle s_{i}\right\rangle\right)$ is the probability that the spin is directed downwards. Equation (13) gives the probability of the presence of a bond in each of the states of the system. For example, the probability of the first and the second spin of the ferromagnetic bond being directed upwards is $\psi_{+}(\uparrow)\left(\mathrm{e}^{K+B} / \lambda_{+}\right) \psi_{+}(\uparrow)$.

Let us consider a finite system made of one spin and one bond. Such a system must be described by the higher and the lower eigenvalue of the matrix $\boldsymbol{F}$. 
The sum over the states or the partition function can be written as

$$
\begin{gathered}
\langle\boldsymbol{\eta}|\boldsymbol{F}| \boldsymbol{\eta}\rangle=\left(\left\langle\boldsymbol{\psi}_{+}\left|+\left\langle\boldsymbol{\psi}_{-}\right|\right) \boldsymbol{F}\left(\left|\boldsymbol{\psi}_{+}\right\rangle+\left|\boldsymbol{\psi}_{-}\right\rangle\right)=\left\langle\boldsymbol{\psi}_{+}|\boldsymbol{F}| \boldsymbol{\psi}_{+}\right\rangle+\left\langle\boldsymbol{\psi}_{-}|\boldsymbol{F}| \boldsymbol{\psi}_{-}\right\rangle\right.\right. \\
=\lambda_{+}+\lambda_{-}=2 \mathrm{e}^{K} \cosh B=\mathrm{e}^{K+B}+\mathrm{e}^{K-B} .
\end{gathered}
$$

In order to determine the magnetisation we look for

$$
\frac{\partial}{\partial B}\langle\boldsymbol{\eta}|\boldsymbol{F}| \boldsymbol{\eta}\rangle=\mathrm{e}^{K+B}-\mathrm{e}^{K-B}
$$

and hence,

$$
\left\langle s_{i}\right\rangle=\frac{\frac{\partial}{\partial B}\langle\boldsymbol{\eta}|\boldsymbol{F}| \boldsymbol{\eta}\rangle}{\langle\boldsymbol{\eta}|\boldsymbol{F}| \boldsymbol{\eta}\rangle}=\frac{\mathrm{e}^{K+B}-\mathrm{e}^{K-B}}{\mathrm{e}^{K+B}+\mathrm{e}^{K-B}} .
$$

When $N \rightarrow \infty$, the problem is simplified as

$$
\begin{aligned}
& \left\langle\boldsymbol{\eta}\left|\boldsymbol{F}^{N}\right| \boldsymbol{\eta}\right\rangle=\left\langle\boldsymbol{\psi}_{+}\left|\boldsymbol{F}^{N}\right| \boldsymbol{\psi}_{+}\right\rangle+\left\langle\boldsymbol{\psi}_{-}\left|\boldsymbol{F}^{N}\right| \boldsymbol{\psi}_{-}\right\rangle \\
& =\lambda_{+}^{N}+\lambda_{-}^{N}=\lambda_{+}^{N}\left[1+\left(\lambda_{-} / \lambda_{+}\right)^{N}\right] \underset{N \rightarrow \infty}{\longrightarrow} \lambda_{+}^{N} .
\end{aligned}
$$

Therefore, we can describe the system not by the partition function but in terms of probabilities as

$$
\left\langle\boldsymbol{\eta}\left|\boldsymbol{F}^{N}\right| \boldsymbol{\eta}\right\rangle=\left\langle\boldsymbol{\psi}_{+}\left|\boldsymbol{F}^{N}\right| \boldsymbol{\psi}_{+}\right\rangle
$$

and

$$
\left\langle\boldsymbol{\eta}\left|\left(\boldsymbol{F} / \lambda_{+}\right)^{N}\right| \boldsymbol{\eta}\right\rangle=\left\langle\boldsymbol{\psi}_{+}\left|\boldsymbol{F} / \lambda_{+}\right| \boldsymbol{\psi}_{+}\right\rangle=1 .
$$

For example, the internal energy per spin can be determined by the mean energy of four events

$$
\begin{aligned}
& \langle\boldsymbol{E}\rangle=\frac{1}{\beta}\left\langle\boldsymbol{\psi}_{+}\left|\left[\begin{array}{cc}
(-K-B) \mathrm{e}^{K+B} / \lambda_{+} & K \mathrm{e}^{-K} / \lambda_{+} \\
K \mathrm{e}^{-K} / \lambda_{+} & (-K+B) \mathrm{e}^{K-B} / \lambda_{+}
\end{array}\right]\right| \boldsymbol{\psi}_{+}\right\rangle \\
& =\frac{1}{\beta \lambda_{+}}\left(-K \mathrm{e}^{K} \cosh B-B \mathrm{e}^{K} \sinh B\right. \\
& \left.+\frac{-K \mathrm{e}^{K} \sinh ^{2} B-B \mathrm{e}^{K} \sinh B \cosh B+\mathrm{e}^{-3 K} K}{\sqrt{\sinh ^{2} B+\mathrm{e}^{-4 K}}}\right) .
\end{aligned}
$$

Similarly, the magnetisation per spin can be determined as

$$
\langle s\rangle=\left\langle\boldsymbol{\psi}_{+}\left|\left[\begin{array}{cc}
\mathrm{e}^{K+B} / \lambda_{+} & 0 \\
0 & -\mathrm{e}^{K-B} / \lambda_{+}
\end{array}\right]\right| \boldsymbol{\psi}_{+}\right\rangle=\frac{\sinh B}{\sqrt{\sinh ^{2} B+\mathrm{e}^{-4 K}}} .
$$




\section{Summary}

The paper presents a generalisation of the method of linear equations over the systems with nonzero temperatures. Two one-dimensional Ising systems have been analysed. For the first model with the integrals $\pm J$ on the square lattice the results have been obtained employing the possibility of reducing the transition matrix to a Markov matrix. In the other model (one-dimensional ferromagnet) the method has been generalised to an arbitrary transition matrix. The next step that still seems possible is the extension of the method to the Ising model $\pm J$ in an external magnetic field. The problem that appears in this method is that the choice of the higher eigenvalue of the transition matrix is equivalent to the assumption that the system is infinite and implies that the averaging is performed on the basis of the states considered. In this model we have two types of states: those of the exchange integrals and those of the spins. The condition of the system infinity should be written in terms of the states $\pm J$, while the averaging should be performed in terms of the spin states at the fixed states $\pm J$.

\section{References}

[1] M. Błaszyk, Acta Phys. Pol. A 104, 3 (2003). 